Vernon H. Neubert

Department of Engineering Science and Mechanics

Pennsylvania State University

University Park, PA 16802

\section{Sensitivity of Eigenvalues to Nonsymmetrical, Dissipative Control Matrices}

\begin{abstract}
Dissipation of energy in vibrating structures can be accomplished with a combination of passive damping and active, constant gain, closed loop control forces. The matrix equations are $\mathbf{M} \ddot{\mathbf{z}}+\mathbf{C} \dot{\mathbf{z}}+\mathbf{K z}=-\mathbf{G} \dot{\mathbf{z}}$. With conventional viscous damping, the damping force is proportional to relative velocity, with $F_{i}=C_{i i} \dot{z}_{i}-C_{i j} \dot{z}_{j}$, where $C_{i i}=C_{i j}$ but the subscripts show the position of the number in the $\mathbf{C}$ matrix. For a dashpot connected directly to ground, $F_{i}=C_{i i} \dot{z}_{i}$. Thus there is a definite pattern to the positions of numbers in the ith and jth rows of the $\mathbf{C}$ matrix, that is, a positive number on the diagonal is paired with an equal negative number, or zero, off the diagonal. With the control matrix $\mathbf{G}$, it is here assumed that the positioning of individual controllers and sensors is flexible, with $F_{i}=G_{i i} \dot{z}_{i}$ or $F_{i}=G_{i j} \dot{z}_{j}$, the latter meaning that the control force at $i$ is proportional to the velocity sensed at $j$. Thus the problem addressed herein is how the individual elements in the $\mathbf{G}$ matrix affect the modal eigenvalues.

Two methods are discussed for finding the sensitivities, the classical method based on the products of eigenvectors and a new method, derived during the present study, involving the derivatives of the invariants in the similarity transformation. Examples are presented for the sensitivities of the complex eigenvalues of the form $\lambda_{r}=-\zeta_{r} \omega_{r}+$ $i \omega_{D r}$ to individual elements in the $\mathbf{G}$ matrix, to combinations of elements, and to a combination of passive damping and active control. Systems with two, three, and eight degrees of freedom are investigated. (C) 1993 John Wiley \& Sons, Inc.
\end{abstract}

\section{INTRODUCTION}

It is sometimes difficult to achieve significant damping in the dominant modes of vibration and then a combination of passive and active damping is desirable. One design philosophy is to achieve as much passive damping as possible or practical and then meet the overall goals by the addition of active control forces that are functions of the measured velocities.

The optimization of viscous damping to minimize free vibrations has been studied by Neubert (1989), Gilheany (1989), Silverberg (1986), McLoughlin (1991), and Inman and Andry (1980), who give additional references. Neubert (1993) also presented an approach for optimum

Shock and Vibration, Vol. 1, No. 2, pp. 153-160 (1993) (C) 1993 John Wiley \& Sons, Inc. sizing and location of viscous damping to minimize forced, random vibrations. Methods of active control are summarized in the book by Meirovitch (1990). The need for passive damping in feedback controlled flexible structures was emphasized by von Flotow and Vos (1990). Venkayya and Tischler (1985) discussed the effect of frequency control on the dynamic response of structures. Sensitivity analysis as related to modal control is the subject of the book by Porter and Crossley (1972) that summarized the methods that were developed up to that time and presented some practical examples for aircraft stabilization systems, cascaded vehicles, and economic and manufacturing systems. Of special interest are the sections dealing with sys-

CCC 1070-9622/93/020153-08 
tems that have confluent, or repeating, eigenvalues rather than distinct eigenvalues with linearly independent eigenvectors, as assumed herein.

The purpose of the present paper is to summarize analytical relationships for the sensitivity of modal damping ratios $\zeta_{r}$ and natural frequencies $\omega_{r}$ to elements in the $\mathbf{G}$, or active control, matrix with specific results for systems with one, two, and eight degrees of freedom. These sensitivities are found by first finding the derivatives of the eigenvalues $\lambda_{r}$. It is assumed that the system is underdamped and the eigenvalues occur in complex conjugate pairs, namely, $\lambda_{r}, \bar{\lambda}_{r}=-\zeta_{r} \omega_{r} \pm$ $i \omega_{D r}$. Having the derivative of this pair of eigenvalues with respect to input parameters, the derivatives of the modal damping ratios $\zeta_{r}$ and natural frequencies $\omega_{r}$ are readily found.

The eigenvalue derivatives are determined in two ways. In the first method, which was developed during the present study, the eigenvalue derivatives are formulated from the invariants in the similarity transformation. Because the input parameters and the eigenvalues appear explicitly, these derivatives aid greatly in understanding the interaction. The second method, which is well known and more convenient for numerical computations, is to take the derivative of the characteristic equation. The derivative then involves the eigenvectors and the derivative of the characteristic matrix.

The matrix equations are

$$
\mathbf{M z}+\mathbf{C} \dot{\mathbf{z}}+\mathbf{K z}=-\mathbf{G} \dot{\mathbf{z}}-\mathbf{H z}
$$

where the $n \times n$ matrices $\mathbf{M}, \mathbf{C}$, and $\mathbf{K}$ are symmetric but $\mathbf{G}$ and $\mathbf{H}$ may be unsymmetric. Equation (1) is multiplied by the inverse of $\mathbf{M}$ to produce

$$
\ddot{\mathbf{z}}+\mathbf{C}_{1} \dot{\mathbf{z}}+\mathbf{K}_{1} \mathbf{z}=-\mathbf{M}^{-1}[\mathbf{G} \dot{\mathbf{z}}+\mathbf{H z}]
$$

where $\mathbf{C}_{1}=\mathbf{M}^{-1} \mathbf{C}$ and $\mathbf{K}_{1}=\mathbf{M}^{-1} \mathbf{K}$. The matrices $\mathbf{C}_{1}$ and $\mathbf{K}_{1}$ are usually unsymmetric. The resulting equations are written in state space form as

$$
\mathbf{I} \dot{\mathbf{y}}(t)-\mathbf{A y}(t)=\mathbf{0}
$$

where

$$
\mathbf{A}=\left[\begin{array}{cc}
-\mathbf{M}^{-1}[\mathbf{C}+\mathbf{G}] & -\mathbf{M}^{-1}[\mathbf{K}+\mathbf{H}] \\
\mathbf{I} & \mathbf{0}
\end{array}\right] .
$$

The solutions of the homogeneous equations are of the form

$$
\mathbf{y}(t)=e^{\lambda t} \mathbf{u},
$$

and the eigenvalue problem to be solved is

$$
[-\lambda \mathbf{I}+\mathbf{A}] \mathbf{u}=\mathbf{0} .
$$

From Eq. (6) the eigenvalues $\lambda_{r}$ are determined and they are here assumed to be distinct, with no repeated eigenvalues. The $r$ th right eigenvector is $\mathbf{u}_{r}$, and it satisfies the relationship

$$
\left[-\lambda_{r} \mathbf{I}+\mathbf{A}\right] \mathbf{u}_{r}=\mathbf{0} .
$$

Because $\mathbf{A}$ is unsymmetric, there is also a set of left eigenvectors associated with the eigenvector problem involving $\mathbf{A}^{\mathbf{T}}$, which is the transpose of A. The eigenvalues $\lambda_{r}$ are the same as in Eq. (7), but the left eigenvectors $\mathbf{v}_{r}$ satisfy

$$
\left[-\lambda_{r} \mathbf{I}+\mathbf{A}^{\mathbf{T}}\right] \mathbf{v}_{r}=\mathbf{0},
$$

which can also be written in transpose form

$$
\mathbf{v}_{r}^{T}\left[-\lambda_{r} \mathbf{I}+\mathbf{A}\right]=\mathbf{0} .
$$

The orthogonality relationships between left and right eigenvectors are

$$
\mathbf{v}_{r}^{T} \mathbf{u}_{s}=0 \quad \text { for } r \neq s
$$

and

$$
\mathbf{v}_{r}^{T} \mathbf{u}_{r}=1 \quad \text { for } r=s
$$

or, combining (10a) and (10b),

$$
\mathbf{V}^{T} \mathbf{U}=\mathbf{I}
$$

where $\mathbf{U}$ and $\mathbf{V}$ are $2 n \times 2 n$ matrices of right and left eigenvectors. It can be shown that

$$
\mathbf{V}^{T} \mathbf{A} \mathbf{U}=\Lambda
$$

where $\Lambda$ is a diagonal matrix of the eigenvalues.

Here Eq. (10b) is the normalizing condition for the $\mathbf{v}_{r}$ vectors, where the $\mathbf{u}_{r}$ have first been normalized according to

$$
\mathbf{u}_{r}^{T} \mathbf{M}^{*} \mathbf{u}_{r}=b_{r} .
$$




\section{SENSITIVITIES OF EIGENVALUES USING SIMILARITY TRANSFORMATION INVARIANTS}

Some of the effects of individual elements in the control matrix can be anticipated from knowledge of the relationship between the matrix $\mathbf{A}$ and the diagonal eigenvalue matrix $\Lambda$. The matrices $\Lambda$ and $\mathbf{A}$ are similar, in that they are related by a similarity transformation. The invariants are those associated with similarity transformations, meaning that the matrices have the same eigenvalues, their traces are equal, and their determinants have the same value. The equality of the traces relates the system damping parameters to the modal damping ratios and natural frequencies, as follows with $\mathbf{H}=\mathbf{0}$.

$$
\begin{aligned}
\operatorname{Tr}(\mathbf{A}) & =\operatorname{Tr}[-(\mathbf{C}+\mathbf{G})]=\operatorname{Tr}(\Lambda) \\
& =\sum_{r} \lambda_{r}+\bar{\lambda}_{r}=\sum_{r}-2 \zeta_{r} \omega_{r}
\end{aligned}
$$

The fact that the determinants are invariant means that

$$
|\mathbf{A}|=\left|\mathbf{M}^{-1} \mathbf{K}\right|=\omega_{1}^{2} \omega_{2}^{2} \cdots \omega_{N}^{2},
$$

so that, even though the $\omega_{r}^{2}$ s change due to changes in $\mathbf{C}+\mathbf{G}$, their product does not change if the mass and stiffness matrices are not altered.

From Eqs. (12) and (13), relationships for the sensitivities can also be determined by taking derivatives of both sides of the equations with respect to $\mathbf{A}_{i j}$. Thus the following important results are obtained.

$$
\begin{aligned}
\frac{\partial[\operatorname{Tr}(\mathbf{A})]}{\partial \mathbf{A}_{i j}} & =\frac{-\partial[\operatorname{Tr}(\mathbf{C}+\mathbf{G})]}{\partial \mathbf{A}_{i j}} \\
& =\frac{\partial\left(\sum_{r} \lambda_{r}+\bar{\lambda}_{r}\right)}{\partial \mathbf{A}_{i j}}=\frac{\partial\left[\sum_{r}\left(-2 \zeta_{r} \omega_{r}\right)\right]}{\partial \mathbf{A}_{i j}} \\
& \frac{\partial\left|\mathbf{M}^{-1} \mathbf{K}\right|}{\partial \mathbf{A}_{i j}}=\frac{\partial\left(\omega_{1}^{2} \omega_{2}^{2} \cdots \omega_{N}^{2}\right)}{\partial \mathbf{A}_{i j}}
\end{aligned}
$$

Equation (12) is written in detail as Eq. (16) and taking the partial of (16) with respect to $\mathbf{A}_{i i}$ confirms Eq. (33), which is derived separately below.

$$
\sum_{r=1}^{2 n} \lambda_{r}=\sum_{i=1}^{2 n} \mathbf{A}_{i i}=\mathbf{A}_{11}+\mathbf{A}_{22}+\cdots+\mathbf{A}_{2 n 2 n}
$$

and

$$
\sum_{r=1}^{2 n} \frac{\partial \lambda_{r}}{\partial \mathbf{A}_{i i}}=1
$$

\section{Example 1: System with One Degree of Freedom}

For the simple one degree of freedom system with a mass $m$ supported by a spring of stiffness $k$, a dashpot with damping rate $c$, and a control force of $-g \dot{x}$ the $\mathbf{A}$ matrix is

$$
\mathbf{A}=\left[\begin{array}{cc}
-m^{-1}(c+g) & -m^{-1} k \\
1 & 0
\end{array}\right]
$$

and the diagonal eigenvalue matrix is

$$
\Lambda=\left[\begin{array}{cc}
-\zeta_{1} \omega_{1}+i \omega_{D 1} & 0 \\
0 & -\zeta_{1} \omega_{1}-i \omega_{D 1}
\end{array}\right]
$$

The following relationships are obtained by setting the traces and determinants equal,

$$
-m^{-1}(c+g)=-2 \zeta_{1} \omega_{1}
$$

and

$$
m^{-1} k=\zeta_{1}^{2} \omega_{1}^{2}+\omega_{D 1}^{2}=\omega_{1}^{2} .
$$

Taking the partial derivatives of Eqs. (19) and (20) with respect to $g$, the results are

$$
m^{-1}=2\left(\zeta_{1} \frac{\partial \omega_{1}}{\partial g}+\omega_{1} \frac{\partial \zeta_{1}}{\partial g}\right)
$$

and

$$
0=\omega_{1} \frac{\partial \omega_{1}}{\partial g}
$$

From Eq. (22), if $\omega_{1} \neq 0$, then $\partial \omega_{1} / \partial g=0$, which shows that changing $g$ does not result in a change in $\omega_{1}$.

\section{Example 2: System with Two Degrees of Freedom}

If the system had two degrees of freedom, the damping matrix $\mathbf{C}=\mathbf{0}$, and the mass matrix is diagonal, the $\mathbf{A}$ and $\Lambda$ matrices would be $4 \times 4$, as follows. 


$$
\begin{aligned}
\mathbf{A} & =\left[\begin{array}{cc}
-\mathbf{M}^{-1} \mathbf{G} & -\mathbf{M}^{-1} \mathbf{K} \\
\mathbf{I} & \mathbf{0}
\end{array}\right] \\
& =\left[\begin{array}{cccc}
-G_{11} / m_{1} & -G_{12} / m_{1} & -K_{11} / m_{1} & -K_{12} / m_{1} \\
-G_{21} / m_{2} & -G_{22} / m_{2} & -K_{21} / m_{2} & -K_{22} / m_{2} \\
1 & 0 & 0 & 0 \\
0 & 1 & 0 & 0
\end{array}\right]
\end{aligned}
$$

and

$$
\Lambda=\left[\begin{array}{cccc}
\lambda_{1} & 0 & 0 & 0 \\
0 & \bar{\lambda}_{1} & 0 & 0 \\
0 & 0 & \lambda_{2} & 0 \\
0 & 0 & 0 & \bar{\lambda}_{2}
\end{array}\right] .
$$

From the traces and determinants, the following relationships arise

$$
\begin{aligned}
& \left(K_{11} K_{22}-K_{12} K_{21}\right) /\left(m_{1} m_{2}\right)=\lambda_{1} \bar{\lambda}_{1} \lambda_{2} \bar{\lambda}_{2}=\omega_{1}^{2} \omega_{2}^{2} \\
& -G_{11} / m_{1}-G_{22} / m_{2}=-\lambda_{1}-\bar{\lambda}_{1}-\lambda_{2}-\bar{\lambda}_{2} \\
& =-2 \zeta_{1} \omega_{1}-2 \zeta_{2} \omega_{2} .
\end{aligned}
$$

The fact that the eigenvalues must be real or occur in complex conjugate pairs is confirmed by Eq. (26) because the left side is real. Further it shows that if $G_{11}=G_{22}=0$ and $G_{12} \neq 0$, then the system will be unstable since $\zeta_{1}$ and $\zeta_{2}$ must then be real and of opposite signs, with $\omega_{1}$ and $\omega_{2}$ real and positive.

By taking the partial derivative of Eqs. (25) and (26) with respect to $G_{11}$, equations for the sensitivities are obtained.

$$
0=\omega_{1} \frac{\partial \omega_{2}}{\partial G_{11}}+\omega_{2} \frac{\partial \omega_{1}}{\partial G_{11}}
$$

and

$$
\begin{aligned}
1 /\left(2 m_{1}\right)= & \zeta_{1} \frac{\partial \omega_{1}}{\partial G_{11}}+\zeta_{2} \frac{\partial \omega_{2}}{\partial G_{11}} \\
& +\omega_{1} \frac{\partial \zeta_{1}}{\partial G_{11}}+\omega_{2} \frac{\partial \zeta_{2}}{\partial G_{11}}
\end{aligned}
$$

An interesting situation arises according to Eq. (27), namely that a change in $G_{11}$ could produce changes in $\omega_{1}$ and $\omega_{2}$ and if one of them increases, the other must decrease.

\section{SENSITIVITIES OF EIGENVALUES IN TERMS OF EIGENVECTORS}

To find the sensitivity of the eigenvalues $\lambda_{r}$ with respect to a parameter in location $\mathbf{A}_{i j}$, take the partial derivative of Eq. (7) with respect to $\mathbf{A}_{i j}$. The well-known equation (Wittrick, 1962; Rogers, 1970) for solving for this derivative in terms of the eigenvectors and the derivative of $\mathbf{A}$ is given in Eq. (29)

$$
\frac{\partial \lambda_{r}}{\partial \mathbf{A}_{i j}}=\mathbf{v}_{r}^{T} \frac{\partial \mathbf{A}}{\partial \mathbf{A}_{i j}} \mathbf{u}_{r} .
$$

The right-hand side of Eq. (29) is a scalar. Taking the partial of $\mathbf{A}$ with respect to $\mathbf{A}_{i j}$ results in a matrix having unity in the $i j$ position and zeroes for all the other matrix elements. Using $\mathbf{v}_{i r}$ to designate the $i$ th element of the vector $\mathbf{v}_{r}$, Eq. (29) becomes

$$
\frac{\partial \lambda_{r}}{\partial \mathbf{A}_{i j}}=\mathbf{v}_{i r} \mathbf{u}_{j r} .
$$

The $2 n \times 2 n$ sensitivity matrix $\mathbf{S}_{r}$ is now formed as defined in Porter and Crossley (1972), which shows how each element $\mathbf{A}_{i j}$ affects $\lambda_{r}$,

$$
\mathbf{S}_{r}=\left[\frac{\partial \lambda_{r}}{\partial \mathbf{A}_{i j}}\right]=\mathbf{v}_{r} \mathbf{u}_{r}^{T} \quad(r, i, j=1,2, \ldots, 2 n) .
$$

The sums of sensitivity matrices are of interest, and, from Eqs. (31) and (10c), are

$$
\sum_{r=1}^{2 n} \mathbf{S}_{r}=\mathbf{V} \mathbf{U}^{T}=\mathbf{I}
$$

where the $\mathbf{U}$ and $\mathbf{V}$ are $2 n \times 2 n$ matrices made up of the right and left eigenvectors, respectively.

Writing Eq. (32) explicitly for the sum of the diagonal elements of the sensitivity matrices shows a significant result in Eq. (33), which is the same as Eq. (16b).

$$
\sum_{r=1}^{2 n} \mathbf{S}_{i i r}=\sum_{r=1}^{2 n} \frac{\partial \lambda_{r}}{\partial \mathbf{A}_{i i}}=1 \quad \text { for } i=j
$$

For the off-diagonal terms,

$$
\sum_{r=1}^{2 n} \mathbf{S}_{i j r}=\sum_{r=1}^{2 n} \frac{\partial \lambda_{r}}{\partial \mathbf{A}_{i i}}=\mathbf{0} \text { for } i \neq j .
$$


The product of two sensitivity matrices for two different eigenvalues is zero, as can be seen from the following, which follow from Eqs. (10).

$$
\begin{aligned}
& \mathbf{S}_{r} \mathbf{S}_{s}=\mathbf{v}_{r} \mathbf{u}_{r}^{T} \mathbf{v}_{s} \mathbf{u}_{s}^{T}=0 \quad(r \neq s) \\
& \mathbf{S}_{r} \mathbf{S}_{r}=\mathbf{v}_{r} \mathbf{u}_{r}^{T} \mathbf{v}_{r} \mathbf{u}_{r}^{T}=\mathbf{S}_{r} \quad(r=s) .
\end{aligned}
$$

If the system is underdamped, the eigenvalues occur in complex conjugate pairs, and the following relationships apply:

$$
\begin{gathered}
\lambda_{r}=-\zeta_{r} \omega_{r}+i \omega_{r}\left(1-\zeta_{r}^{2}\right)^{\frac{1}{2}}=-\zeta_{r} \omega_{r}+i \omega_{D r} \\
\lambda_{r} \bar{\lambda}_{r}=\omega_{r}^{2} \quad \text { and } \quad \lambda_{r}+\bar{\lambda}_{r}=-2 \zeta_{r} \omega_{r}
\end{gathered}
$$

If there are $2 n$ eigenvalues, there are only $n$ different modal frequencies $\omega_{r}$. The $\omega_{D r}$ is the modal frequency of damped free vibrations.

By differentiating the two expressions in Eq. (36b) with respect to $\mathbf{A}_{i j}$, the derivatives of the modal damping ratio and natural frequency, that is $\partial \zeta_{r} / \partial \mathbf{A}_{i j}$ and $\partial \omega_{r} / \partial \mathbf{A}_{i j}$, are readily determined in terms of the derivatives of $\lambda_{r}$ and $\bar{\lambda}_{r}$.

\section{Example 3: Ten-Bar Truss with Eight Degrees of Freedom}

The ten-bar truss studied (Fig. 1), is one that has been suggested by some investigators as one of a group of "standard" trusses and was used as an example in Neubert $(1989,1993)$. It is not necessarily a practical truss, but is used for comparison purposes. The truss lies in the $x-y$ plane and has four moving nodal points, numbers $1,2,3$, and 4, which are free to move in the $x$ and $y$ directions. There are two anchored nodal points, numbered 5 and 6 . Each truss bay is square, 360 in. on each side. The cross-sectional areas are given in Table 1. The material of each bar is aluminum with a Young's Modulus, $E=10 \times 10^{6}$

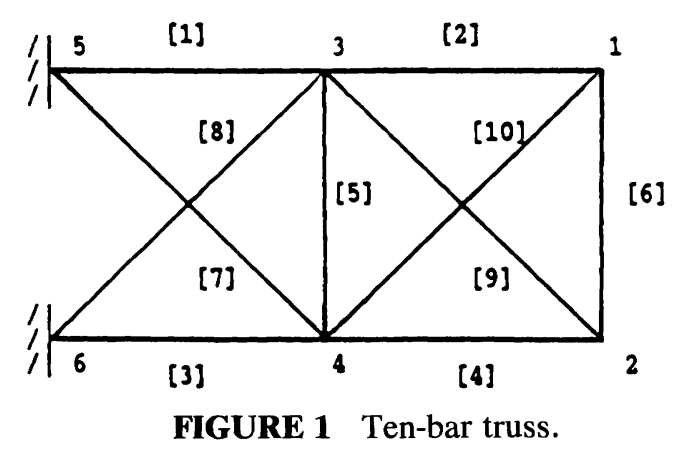

Table 1 Areas of Truss Bars

\begin{tabular}{cccc}
\hline $\begin{array}{c}\text { Bar No. } \\
(\mathrm{m})\end{array}$ & $\begin{array}{c}\left.\text { Area (in. }{ }^{2}\right) \\
\left(A_{\mathrm{m}}\right)\end{array}$ & $\begin{array}{c}\text { Bar No. } \\
(\mathrm{m})\end{array}$ & $\begin{array}{c}\left.\text { Area (in. }{ }^{2}\right) \\
\left(A_{\mathrm{m}}\right)\end{array}$ \\
\hline 1 & 31.5 & 6 & 0.5 \\
2 & 0.1 & 7 & 7.5 \\
3 & 23.0 & 8 & 20.5 \\
4 & 15.5 & 9 & 21.0 \\
5 & 0.1 & 10 & 0.1 \\
\hline
\end{tabular}

psi. Half the mass of each bar was lumped at each end of the bar in the present analysis.

Optimization of passive viscous damping, representable by dashpots connected between nodal points, was presented in Neubert $(1989,1993)$ for this truss. In practice, the control forces represented by the $\mathbf{G} \dot{\mathbf{z}}$ would be superimposed on the damping forces generated by $\mathbf{C} \dot{\mathbf{z}}$. In order to isolate the effects of the control forces, no passive damping is included in these first examples, so the matrix $\mathbf{C}=\mathbf{0}$ initially.

It is assumed that a control force can be located at any of the four moving truss nodes and that it is proportional to velocity at that or any other node, in the $x$ or $y$ direction. The positions in the $\mathbf{G}$ matrix follow the numbering of the nodes on the truss. Thus rows 1,2 , and 3 correspord to $1 x, 1 y$, and $2 x$ on the truss, where $2 x$ means the displacement at node 2 in the $x$ direction. Hence $G_{23}=G_{1 y, 2 x}$ and $G_{57}=G_{3 x, 5 x}$ for example. The sensitivities of the eigenvalues to individual elements in the $G$ matrix were found using Eq. (30). The relationships in Eqs. (14)(16) help to understand and confirm the results, but they were not used directly in the computations of sensitivities.

The modal damping ratios $\zeta_{r}$ of the closed loop system are of particular interest. For this truss, it was found that the damping ratios in different modes are affected by various choices of location and magnitude of $G_{i j}$. In Fig. 2, the damping ratios $\zeta_{6}, \zeta_{5}$, and $\zeta_{8}$ are plotted for both positive and negative values of $G_{55}$. It is seen that for small, positive values of $G_{55}$ the damping ratios $\zeta_{5}$ and $\zeta_{6}$ are both positive and increase almost linearly with $G_{55}$. All the modal damping ratios are positive in this range and the system is stable. For larger values of $G_{55}, \zeta_{6}$ increases at a faster rate and $\zeta_{5}$ decreases, but they remain positive. The curves are antisymmetrical with respect to the origin.

The sensitivities, or derivatives of $\zeta_{5}, \zeta_{6}$, and $\zeta_{8}$ with respect to $G_{55}$ are shown in Fig. 3. These are 


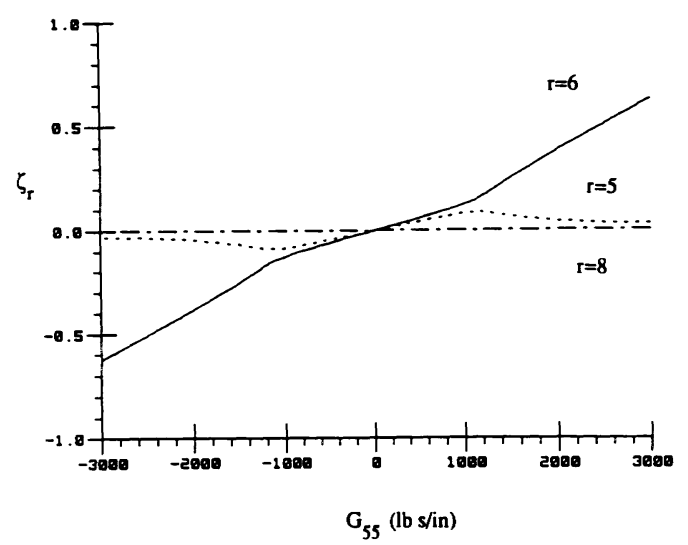

FIGURE 2 Variation of $\zeta_{6}, \zeta_{5}$, and $\zeta_{8}$ with $G_{55}$.

related directly to the slopes of the curves in Fig. 2. When the sensitivity is positive, the damping ratio is increasing. Thus for $\zeta_{6}$, the slope is always positive in the range of interest, and for $\zeta_{5}$ the slope is positive when the absolute value of $G_{55}$ is small but negative for larger values of $G_{55}$.

The damping ratios of the same three modes are shown in Fig. 4 versus $G_{77}$. The corresponding natural frequencies $\omega_{r}$ are shown in Fig. 5 and the damped natural frequencies $\omega_{D r}$ in Fig. 6 versus $G_{77}$. It is seen that damping is highest in the eighth mode and that $\zeta_{8}$ approaches 1.0 or critical damping as $G_{77}$ becomes large, but $\zeta_{5}$ and $\zeta_{6}$, as well as the damping ratios for the other five modes, remain relatively small. The range of the natural frequencies for small damping is from 131 to $796 \mathrm{rad} / \mathrm{s}$. When $\zeta_{8}=1.0$, the damped natural frequency $\omega_{D 8}=0$ and the modal frequency $\omega_{8}=$ $598 \mathrm{rad} / \mathrm{s}$, so there is a decrease of about $25 \%$ in $\omega_{8}$. At the same time the other seven modes all show increases of $0.03-7.5 \%$ for their $\omega_{r}$ 's.

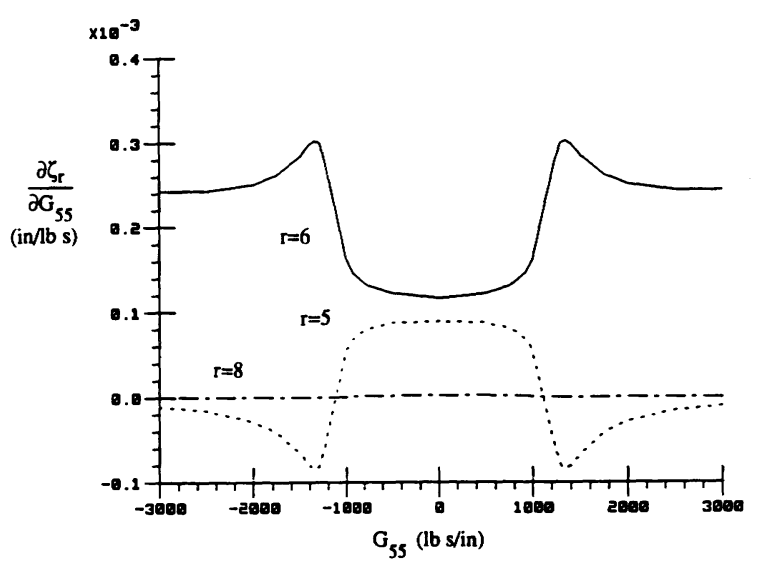

FIGURE $3 \partial \zeta_{r} / \partial G_{55}$ versus $G_{55}$ for $r=6,5$, and 8 .

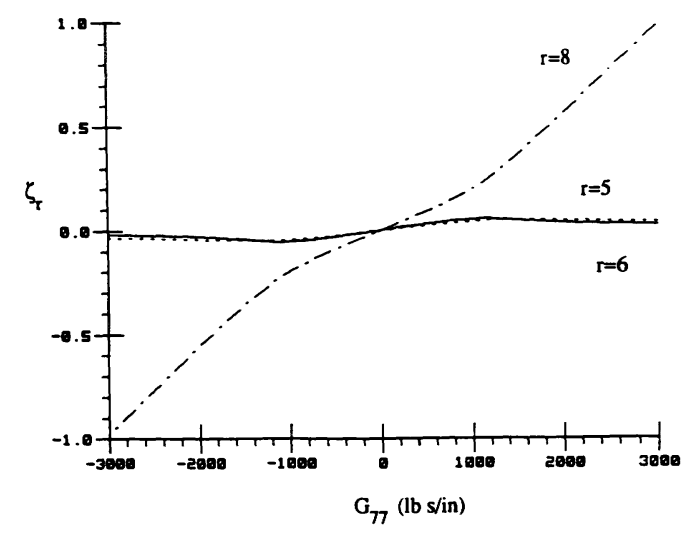

FIGURE $4 \zeta_{5}, \zeta_{6}$, and $\zeta_{8}$ versus $G_{77}$.

It should be mentioned that it becomes difficult to trace the modes as the modal damping ratio increases. The mode numbers are here decided in association with small control forces. If the modes are ordered according to the magnitude of $\omega_{D r}$, the damped natural frequency, then what was the eighth mode for small control forces becomes the first, or lowest frequency, mode for large control forces.

In Fig. 7, the damping ratios are plotted for an input control force for $G_{75}$. The sensitivities are not plotted, but are readily visualized as the slope of the $\zeta_{r}$ curves. The $\zeta_{5}$ and sensitivities are positive for positive $G_{75}$ but $\zeta_{6}$ shows a negative, decreasing trend in the region. Thus the system is unstable if only the $G_{75}$ control force is applied. If it is desired to increase the damping in modes 5 and 8 and decrease that in mode 6 , then a positive $G_{75}$ should be used. A negative $G_{75}$ would produce the reverse effect. The effect of $G_{57}$ on the modal damping ratios is similar to that of $G_{75}$.

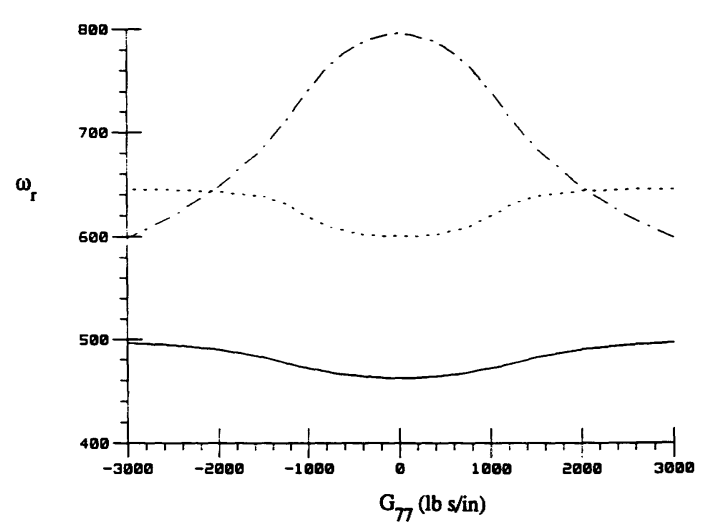

FIGURE $5 \omega_{5}, \omega_{6}$, and $\omega_{8}$ versus $G_{77}$. 


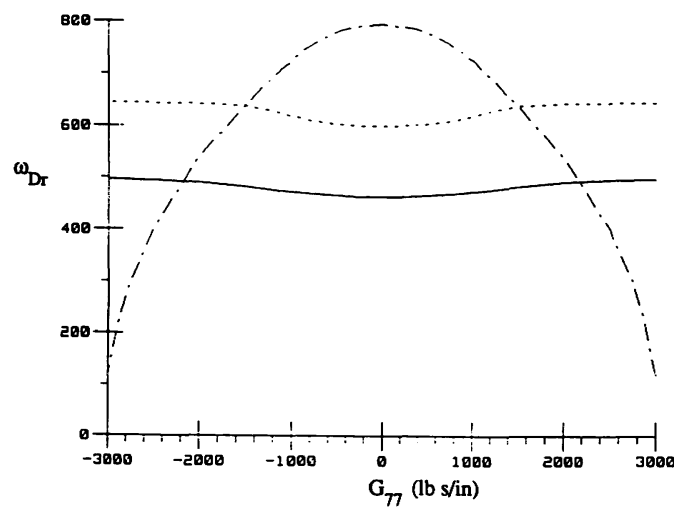

FIGURE $6 \omega_{D 5}, \omega_{D 6}$, and $\omega_{D 8}$ versus $G_{77}$.

The relationships between $\zeta_{r}$ and $G_{i i}$ or $G_{i j}$ are nonlinear. The plots show a nearly linear range only for small control forces. These nonlinearities are due to changes in mode shapes. Large viscous damping or velocity-proportional control forces tend to stiffen the structure and change the mode shapes. The eigenvectors are, in general, complex and their sensitivities are readily calculated, but that is beyond the scope of this article.

Because of the nonlinear behavior, the question arises as to how well the effects of $G_{55}, G_{75}$, $G_{57}$, and $G_{77}$ superimpose. This is answered graphically in the plots in Fig. 8, where $\zeta_{6}$ is shown for the four control forces applied simultaneously, with $G_{55}$ and $G_{77}$ positive but with $G_{75}$ and $G_{57}$ negative, to increase $\zeta_{6}$. For the dashed line, the separate effects were added; for the solid line, the forces were applied simultaneously. It is clear that superposition applies for small control forces only. Note that the range of the control force in Fig. 8 is much smaller than in

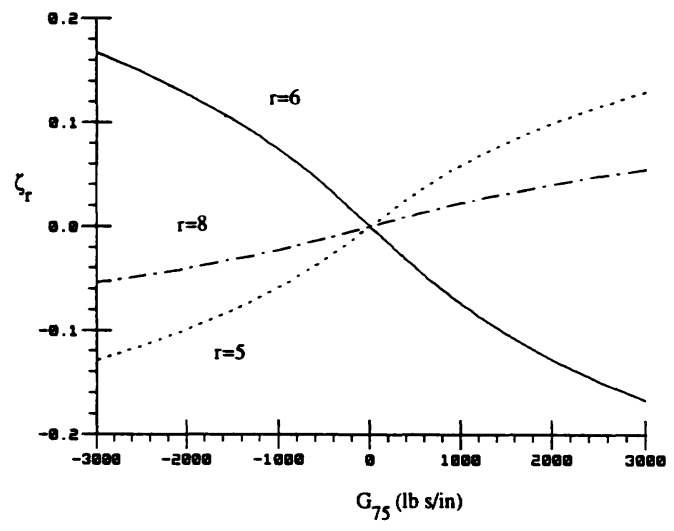

FIGURE $7 \zeta_{5}, \zeta_{6}$, and $\zeta_{8}$ versus $G_{75}$.

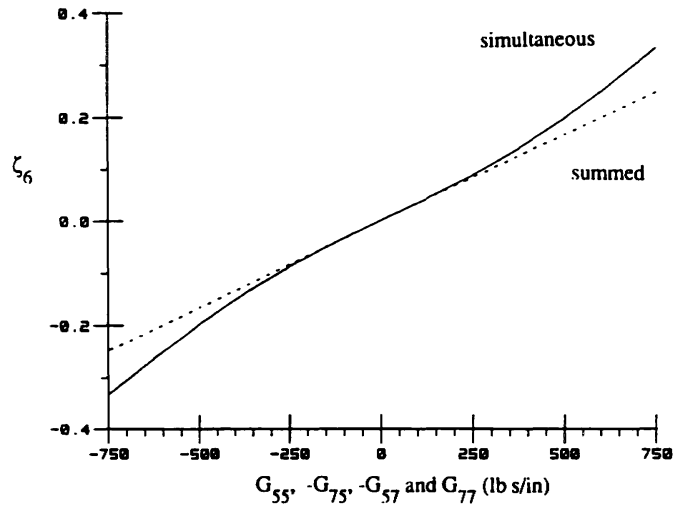

FIGURE $8 \zeta_{6}$ with effects of $G_{55},-G_{75},-G_{57}$, and $G_{77}$ applied simultaneously (-) and superimposed (---).

the previous figures. As the magnitude of the four control forces, applied simultaneously, approaches $3000 \mathrm{lb}$. s/in., the value of $\zeta_{6}=1.77$ and the damping ratios of all the other modes are less than 0.1 , so mode 6 usurps the dissipation.

\section{Example 4: Effect of Initial Passive Damping, 10-Bar Truss}

Passive viscous damping, which is representable mathematically by dashpots connected in parallel to each truss member, tends to produce dissipation in every mode of vibration and the system is stable. If there is passive damping and only one active control force is added in an off-diagonal position, such as $G_{18}$, then the resulting motion can be stable or unstable, depending on the relative magnitudes of the passive and active forces. Using the 10-bar truss as an example, proportional damping was included by having a dashpot parallel to each truss member, so the $C=\beta K$, with $\beta=3.1623 \times 10^{-4} \mathrm{~s}$. Then the value of $G_{18}$ was varied from -200 to $200 \mathrm{lb} / \mathrm{in}$. s. The result is shown in Fig. 9 as a plot of $\zeta_{2}$ and $\zeta_{4}$ versus $G_{18}$. The damping for the other six modes are not small, but they are affected little by $G_{18}$. The solid line is for the system with both viscous damping and the single control force. The dotdash line represents the values when the viscous damping is zero and only the single control force is active. For the latter situation, the trace of $\mathbf{C}+$ $\mathbf{G}$ is zero, so $\zeta_{2}$ and $\zeta_{4}$ tend to have opposite signs and the system is unstable. When the passive damping is added, the curves shift upward and the system becomes more stable. 


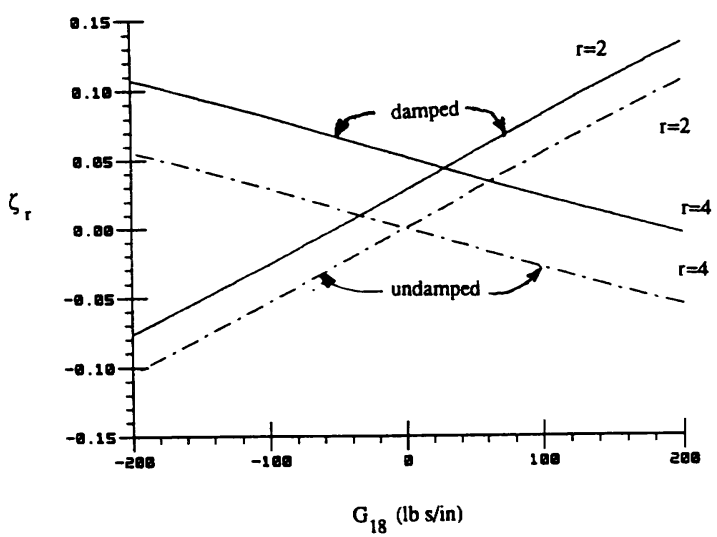

FIGURE $9 \zeta_{2}$ and $\zeta_{4}$ versus $G_{18}$ with and without proportional passive damping.

\section{CONCLUSIONS}

1. A new method for finding eigenvalue sensitivities is presented, based on derivatives of the invariants associated with the similarity transformations involved in the eigenvalue solutions.

2. It is demonstrated that increasing damping may sometimes be deleterious, because some modes may usurp, or "hog," the damping with the result that damping ratios actually decrease for other modes.

3. The modal damping ratios $\zeta_{r}$, are odd functions of the $G_{i j}$, so their sensitivities $\partial \zeta_{r} / \partial G_{i j}$ are even functions. If there is no passive damping, the $\zeta_{r}$ tend to be positive when the diagonal elements $G_{i i}$ are positive. However, the $\zeta_{r}$ can be positive or negative when the off-diagonal elements $G_{i j}$ are positive.

4. The $\zeta_{r}$ are nonlinear functions of the various $G_{i j}$. For small control forces, the relationship is nearly linear. The nonlinearity for larger $G_{i j}$ is due to changes in mode shapes.

5. In view of the changes in mode shapes, complex eigenvectors must be used and proportional damping cannot be assumed except for small $\zeta_{r}$.

6. The study emphasized the effects of individual elements $G_{i j}$ in the $\mathbf{G}$ matrix. If combinations of the $G_{i j}$ are used, corresponding to multiple control forces, their individual effects superimpose accurately only for small control forces. This is because the changes in mode shapes for individual $G_{i j}$ are not the same as for combinations of $G_{i j}$.

7. As has been well known previously, the addition of passive damping tends to have a stabilizing effect in combination with the types of control forces considered herein.

\section{REFERENCES}

Gilheany, J. J., 1989, “'Optimal Selection of Dampers for Freely Vibrating Multidegree of Freedom Systems," In Proceedings of Damping '89, Flight Dynamics Laboratory of the Air Force Wright Aeronautical Laboratories, pp. FCC-1-FCC-18.

Inman, D. J., and Andry, A. N., Jr., 1980, “A Procedure for Designing Overdamped Lumped Parameter Systems," Shock and Vibration Bulletin, Vol. 54, pp. 49-53.

McLoughlin, F. A., 1991, Integrated Structural Damping and Control System Design for High-Order Flexible Systems, Ph.D. Dissertation, Stanford University.

Meirovitch, L., 1990, Dynamics and Control of Structures, Wiley, New York.

Neubert, V. H., 1989, "'Optimization of Energy Dissipation Rate in Structures," In Proceedings of Damping '89, Flight Dynamics Laboratory of the Air Force Wright Aeronautical Laboratories, pp. FCD-1-FCD-24.

Neubert, V. H., 1993, “Optimization of Location and Amount of Viscous Damping to Minimize Random Vibration," Journal of the Acoustical Society of America, Vol. 93, pp. 2707-2715.

Porter, B., and Crossley, R., 1972, Modal Control, Barnes \& Noble Books, New York.

Rogers, L. C., 1970, "Derivatives of Eigenvalues and Eigenvectors,' AIAA Journal, Vol. 8, pp. 943-944.

Silverberg, L., 1986, "Uniform Damping Control of Spacecraft," Journal of Guidance, Control, Dynamics, Vol. 9, pp. 221-227.

Venkayya, V. B., and Tischler, V. A., 1985, "Frequency Control and Its Effect on the Dynamic Response of Flexible Structures," AIAA Journal, Vol. 23, pp. 1768-1774.

von Flotow, A. H., and Vos, D. W. 1991, "The Need for Passive Damping in Feedback Controlled Flexible Structures," In Proceedings of Damping '91, Flight Dynamics Laboratory of the Air Force Wright Aeronautical Laboratories, pp. GBB-1GBB-12.

Wittrick, W. H. 1965, "Rates of Change of Eigenvalues, with Reference to Buckling and Vibration Problems," Proceedings of the Royal Society, Vol. 66, pp. 590-591. 

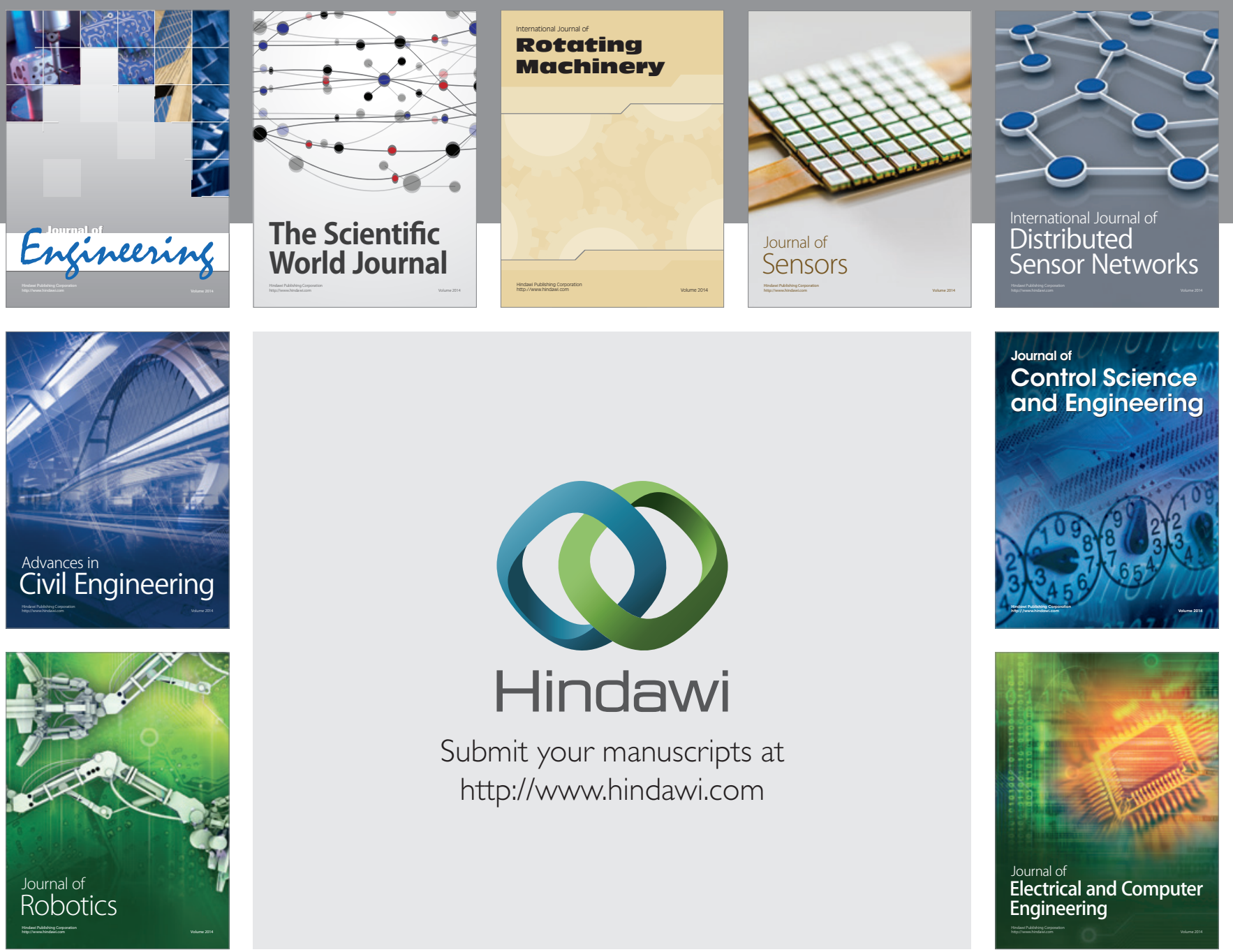

Submit your manuscripts at

http://www.hindawi.com
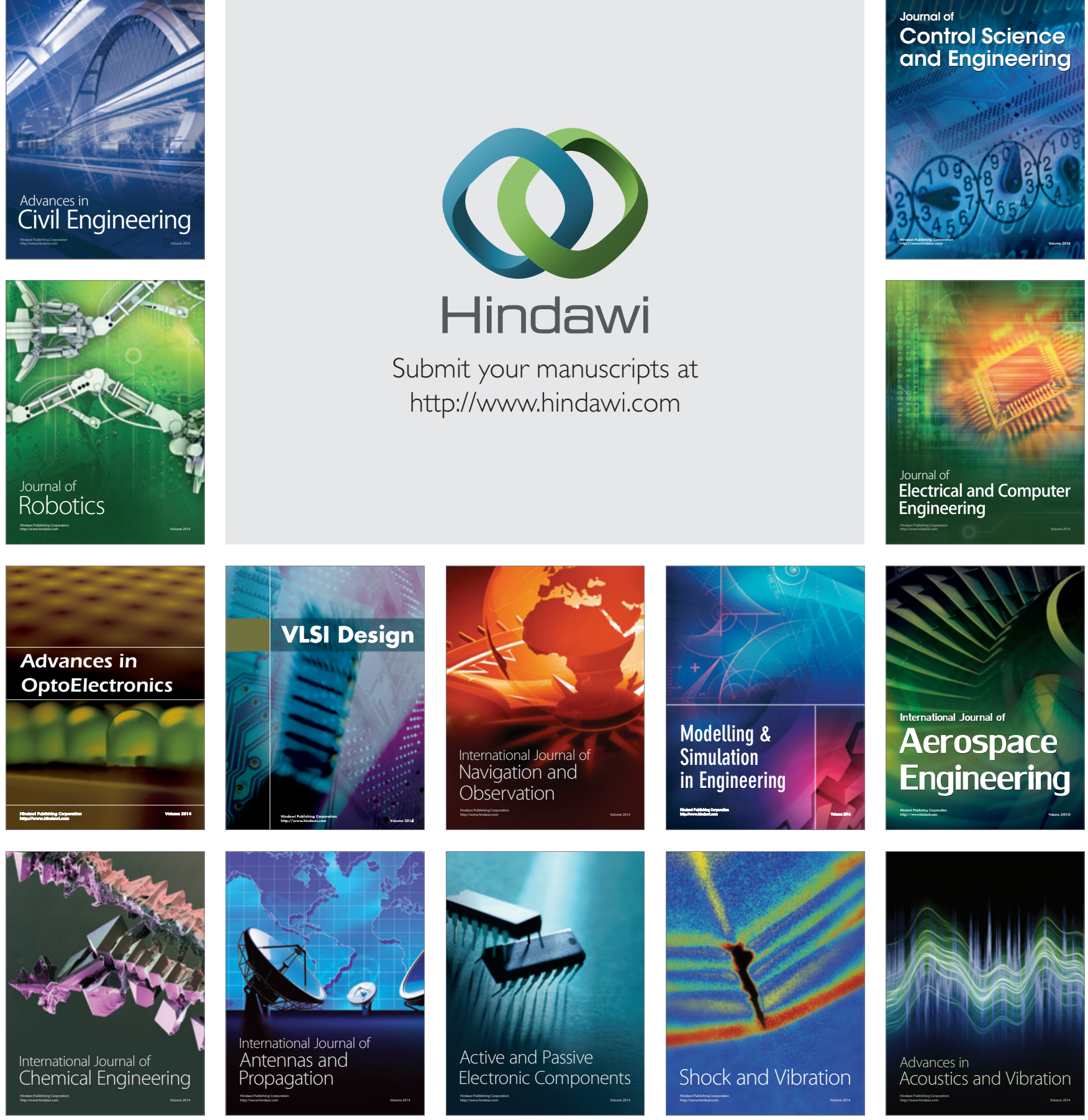